Louisiana State University

LSU Digital Commons

Faculty Publications

Department of Physics \& Astronomy

8-27-2014

\title{
Compressive direct measurement of the quantum wave function
}

Mohammad Mirhosseini

University of Rochester Institute of Optics

Omar S. Magaña-Loaiza

University of Rochester Institute of Optics

Seyed Mohammad Hashemi Rafsanjani

University of Rochester

Robert W. Boyd

University of Rochester Institute of Optics

Follow this and additional works at: https://digitalcommons.Isu.edu/physics_astronomy_pubs

\section{Recommended Citation}

Mirhosseini, M., Magaña-Loaiza, O., Hashemi Rafsanjani, S., \& Boyd, R. (2014). Compressive direct measurement of the quantum wave function. Physical Review Letters, 113 (9) https://doi.org/10.1103/ PhysRevLett.113.090402

This Article is brought to you for free and open access by the Department of Physics \& Astronomy at LSU Digital Commons. It has been accepted for inclusion in Faculty Publications by an authorized administrator of LSU Digital Commons. For more information, please contact ir@lsu.edu. 


\title{
Compressive Direct Measurement of the Quantum Wave Function
}

\author{
Mohammad Mirhosseini, ${ }^{1, *}$ Omar S. Magaña-Loaiza, ${ }^{1}$ Seyed Mohammad Hashemi Rafsanjani, ${ }^{2}$ and Robert W. Boyd ${ }^{1,3}$ \\ ${ }^{1}$ The Institute of Optics, University of Rochester, Rochester, New York 14627, USA \\ ${ }^{2}$ Center for Coherence and Quantum Optics and the Department of Physics and Astronomy, \\ University of Rochester, Rochester, New York 14627, USA \\ ${ }^{3}$ Department of Physics, University of Ottawa, Ottawa, Ontario K1N 6N5, Canada
}

(Received 13 May 2014; published 27 August 2014)

\begin{abstract}
The direct measurement of a complex wave function has been recently realized by using weak values. In this Letter, we introduce a method that exploits sparsity for the compressive measurement of the transverse spatial wave function of photons. The procedure involves weak measurements of random projection operators in the spatial domain followed by postselection in the momentum basis. Using this method, we experimentally measure a 192-dimensional state with a fidelity of $90 \%$ using only 25 percent of the total required measurements. Furthermore, we demonstrate the measurement of a 19200-dimensional state, a task that would require an unfeasibly large acquiring time with the standard direct measurement technique.

DOI: 10.1103/PhysRevLett.113.090402

PACS numbers: 03.65.Ta, 03.65.Wj, 42.15.Dp, 42.50.Ex
\end{abstract}

The no-cloning theorem prohibits the exact determination of the quantum wave function from a single measurement [1-3]. In contrast, a large ensemble of identically prepared quanta can be used to estimate the wave function through quantum state tomography (QST) [4-12]. QST requires the measurement of $d^{2}-1$ independent real numbers for characterizing a $d$-dimensional system. The rapid growth in the number of the required measurements along with the time-consuming postprocessing make QST increasingly more challenging as $d$ grows $[9,13]$.

Recently, there has been considerable interest in determining the wave function of a pure quantum state through the use of weak values [14-19]. This method, known as the direct measurement (DM) method, provides a convenient procedure for estimation of a wave function. It has been suggested that the DM method is an efficient means for characterizing high-dimensional states due to the simplicity of its realization and the absence of a timeconsuming postprocessing [14]. Yet, the measurement of high-dimensional states remains a challenging task. Even for the DM method the number of measurements that are needed to characterize the state vectors grows linearly with the dimension of the state. Further, a much larger ensemble of identically prepared particles is required for the reliable measurement of elements of the state vector in a highdimensional Hilbert space [20].

In this Letter, we introduce a method that combines the benefits of the direct measurement method with a novel computational technique known as compressive sensing [21-26]. Utilizing our approach, the wave function of a high-dimensional state can be estimated with a high fidelity using a much smaller number of measurements than the standard direct measurement approach. In the following we first briefly discuss the direct measurement method and then propose the compressive direct measurement (CDM) method. We then describe our experimental implementation of the CDM method. In our experiment, we were able to reconstruct a wave function with only a fraction of the required measurements for a direct measurement with a more than 90 percent fidelity.

A weak value is the expectation value of a weak measurement followed by a postselection [27]. Consider a weak measurement of the position operator $\hat{\pi}_{j}=\left|x_{j}\right\rangle\left\langle x_{j}\right|$ at point $x_{j}$ followed by a postselection on the zero momentum eigenstate $|o\rangle$. The weak value for the above measurement is given by

$$
\pi_{w}=\frac{\left\langle o \mid x_{j}\right\rangle\left\langle x_{j} \mid \psi\right\rangle}{\langle o \mid \psi\rangle}=\frac{\psi\left(x_{j}\right)}{\phi_{0} \sqrt{N}}
$$

In deriving this formula we have used the Fourier transform property $\phi_{0}=\langle o \mid \psi\rangle$, where $N$ is the dimension of the Hilbert space. We also treat $\phi_{0}$ as a real number as this can always be accounted for by adding a constant phase to the wave function. The above relation indicates that the wave function can be extracted from the weak value, whose real and imaginary parts can be read from the expectation values of two conjugate variables of the pointer (see below) [28]. Note that the transition from the continuous spatial domain to a discrete state vector has been achieved by dividing the continuous coordinate into a finite number of pixels of sufficiently small area.

We now generalize the formalism to a form suitable for compressive sensing. A quantum measurement can be mathematically described by the coupling between the measured system and the measurement pointer. We utilize a two-level pointer such as the polarization of photons. Let the initial system-pointer state be 


$$
|\Omega\rangle=|\psi\rangle \otimes|V\rangle=\sum_{i=1}^{N} \psi_{i}\left|x_{i}\right\rangle \otimes|V\rangle .
$$

Here, $|\psi\rangle$ is the wave function of interest. We have assumed that the pointer is initially prepared in the vertical eigenstate $|V\rangle$. Consider a weak measurement of the sensing operator $\hat{Q}_{m}=\sum_{j} Q_{m, j} \hat{\pi}_{j}$, which is a linear combination of the position projectors $\hat{\pi}_{j}$ weighted with coefficients $Q_{m, j} \in \mathbb{R}$. The effect of this measurement can be described by making a Taylor series approximation to the measurement's evolution operator

$$
e^{i \alpha \hat{Q}_{m} \otimes \hat{\sigma}_{y}}|\Omega\rangle \simeq|\Omega\rangle+\alpha \sum_{j} Q_{m, j} \psi_{j}\left|x_{j}\right\rangle \otimes|H\rangle .
$$

Here, $\hat{\sigma}_{y}=-i|H\rangle\langle V|+i| V\rangle\langle H|$ is a Pauli matrix and $\alpha$ is a constant parameter quantifying the weakness of the measurement. Note that the polarization at each point $x_{j}$ is rotated around the optical axis by the value $Q_{m, j} \alpha$.

The state of the pointer after postselection on $|o\rangle$ is given by

$$
\left|s_{m}\right\rangle=|V\rangle+\frac{\alpha}{\phi_{0} \sqrt{N}} \sum_{j} Q_{m, j} \psi_{j}|H\rangle .
$$

At this stage the information about the state vector $\psi_{j}$ is encoded in the polarization of the postselected photons. The expected values of the polarization of the postselected state can be obtained as

$$
\begin{gathered}
\bar{\sigma}_{x, m} \equiv\left\langle s_{m}\left|\hat{\sigma}_{x}\right| s_{m}\right\rangle=\kappa \sum_{j} Q_{m, j} \operatorname{Re}\left[\psi_{j}\right], \\
\bar{\sigma}_{y, m} \equiv\left\langle s_{m}\left|\hat{\sigma}_{y}\right| s_{m}\right\rangle=-\kappa \sum_{j} Q_{m, j} \operatorname{Im}\left[\psi_{j}\right],
\end{gathered}
$$

where $\hat{\sigma}_{x}=|H\rangle\langle V|+| V\rangle\langle H|$ and $\kappa=(2 \alpha) /\left(\phi_{0} \sqrt{N}\right)$. In the above relations, $\operatorname{Re}\left[\psi_{j}\right]$ and $\operatorname{Im}\left[\psi_{j}\right]$ are the real and the imaginary parts of $\psi_{j}$ respectively. Combining the results $\bar{\sigma}_{x, m}$ and $\bar{\sigma}_{y, m}$ to a complex value $\phi_{m}=(1 / \kappa)\left[\bar{\sigma}_{x, m}-i \bar{\sigma}_{y, m}\right]$ and repeating the measurement several times we obtain $\boldsymbol{\phi}=\mathbf{Q} \boldsymbol{\psi}$. The extended form of this equation reads

$$
\left(\begin{array}{c}
\phi_{1} \\
\phi_{2} \\
\vdots \\
\phi_{M}
\end{array}\right)=\left(\begin{array}{cccc}
Q_{1,1} & Q_{1,2} & \cdots & Q_{1, N} \\
Q_{2,1} & Q_{2,2} & \cdots & Q_{2, N} \\
\vdots & \vdots & \ddots & \vdots \\
Q_{M, 1} & Q_{M, 2} & \cdots & Q_{M, N}
\end{array}\right)\left(\begin{array}{c}
\psi_{1} \\
\psi_{2} \\
\vdots \\
\psi_{N}
\end{array}\right) .
$$

Here, $m \in\{1: M\}$ and $n \in\{1: N\}$ where $M$ is the total number of sensing operators and $N$ is the dimension of the Hilbert state of the unknown wave function. To find the wave function $\psi$ we need to solve the above linear system of equations. For the special case $M=N$ the set of equations can be exactly solved for a nonsingular matrix Q. However, we are interested in the case where $M \leq N$. The pseudoinverse of $\mathbf{Q}$ can be used as an optimal linear recovery strategy to find a solution that minimizes the least square error $[29,30]$

$$
\boldsymbol{\psi}=\mathbf{Q}^{\dagger}\left(\mathbf{Q} \mathbf{Q}^{\dagger}\right)^{-1} \boldsymbol{\phi} .
$$

However, a nonlinear strategy can be used to recover $\boldsymbol{\psi}$ with a far superior quality using the idea of compressive sensing. Consider a linear transformation represented by the matrix $\mathbf{T}$. If the wave function under the experiment $\boldsymbol{\phi}$ is known to have very few nonzero coefficients under this transformation, $\boldsymbol{\psi}$ can be recovered by solving the convex optimization problem [29]

$$
\min _{\boldsymbol{\psi}^{\prime}}\left\|\mathbf{T} \boldsymbol{\psi}^{\prime}\right\|_{\ell_{1}}, \quad \text { subject to } \mathbf{Q} \boldsymbol{\psi}^{\prime}=\boldsymbol{\phi},
$$

where $\|\cdot\|_{\ell 1}$ represents the 1-norm. For this approach to work, it is critical that the two bases, defined by $Q$ and $T$, are incoherent [29]. The coherence of the two bases is defined by the square root of the dimension of the bases times the highest fidelity between any pairs of states from the two bases [31]. According to the theory of compressive sensing if the coherence of the two bases is much smaller than unity (dimensionless), by an overwhelming probability, the target wave function $\psi$ can be recovered with $M \geq O[K \log (N)]$ measurements, where $K$ is the number of nonzero components of $\mathbf{T} \boldsymbol{\psi}$ [31]. Functions with spatial correlations are shown to be extremely likely to have sparse coefficients in discrete cosine transform or wavelet transform domains $[29,32]$. However, a much simpler variant of Eq. (9) can be used in practice to achieve results of comparable quality $[29,33]$. In this method the target wave function can be found by minimizing the quantity

$$
\min _{\boldsymbol{\psi}^{\prime}} \sum_{j}\left\|\nabla \boldsymbol{\psi}_{j}^{\prime}\right\|_{\ell_{1}}+\frac{\mu}{2}\left\|\mathbf{Q} \boldsymbol{\psi}^{\prime}-\boldsymbol{\phi}\right\|_{\ell_{2}}^{2} \text {. }
$$

Here, $\nabla \boldsymbol{\psi}_{j}^{\prime}$ is the discrete gradient of $\boldsymbol{\psi}^{\prime}$ at position $x_{j}$ and $\mu$ is a penalty factor. Heuristically, the minimization of the first term results in a smooth function while the second factor minimizes deviations from the experimental results $\phi$. The optimal value of $\mu$ should be chosen considering the specifics of the target wave function and the signal-to-noise ratio of the experimental data. At the end we retrieve the wave function from the solution of the optimization problem as $\left|\psi^{\prime}\right\rangle=\sum_{i=1}^{N} \psi_{i}^{\prime}\left|x_{i}\right\rangle$.

Figure 1 shows the schematics of the experiment. A vertically polarized Gaussian mode is prepared by spatially filtering a He-Ne laser beam with a single mode fiber and passing it through a polarizer. The polarization rotation is performed using a spatial light modulator (SLM) in combination with two quarter wave plates (WP1 and WP2) [34,35]. The SLM provides the ability to rotate 


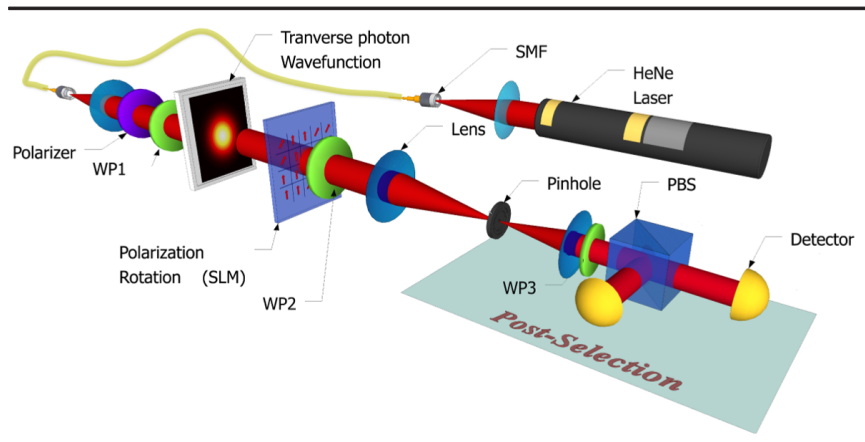

FIG. 1 (color online). A schematic illustration of the experimental setup. The collimated Gaussian beam from a single mode fiber is passed through a polarizer to prepare a vertical polarization. The SLM is used along with two quarter wave plates (WP1 and WP2) to rotate the polarization at each pixel. An $f=50 \mathrm{~cm}$ lens focuses the beam onto a pinhole with a diameter of $10 \mu \mathrm{m}$. The polarization measurement is performed on the light collected from the pinhole using a QWP/HWP (WP3) and a polarizing beam splitter.

the polarization of the incident beam at every single pixel in a controlled fashion. The postselection in the momentum basis is done using a Fourier-transforming lens and a single mode pinhole. We retrieve the real part of the weak value using a combination of a half wave plate (WP3) and a polarizing beam splitter (PBS). The beams from the output ports of the beam splitter are coupled to single mode fibers that are connected to avalanche photodiodes. Similarly, the imaginary part of the weak value is measured by replacing the half wave plate with a quarter wave plate (WP3).

We perform a random polarization rotation of either $20^{\circ}$ or zero at each pixel. The rotated polarization state is given by $\cos \theta|V\rangle+\sin \theta|H\rangle$ where $\sin \theta=Q_{m, j} \alpha$. This corresponds to $Q_{m, j}$ values of 1 and 0 and $\alpha=0.349$. For different values of $m$, we load different pregenerated sensing vectors $\mathbf{Q}_{m}$ onto the SLM and repeat the experiment. The wave function is then retrieved via postprocessing on a computer. We use the algorithm known as the total variation minimization by augmented Lagrangian and alternating direction algorithm [36] to solve Eq. (10). In our experiment, we have used values of $\mu$ ranged from $2^{4}$ to $2^{13}$ (a larger value of $\mu$ results in a closer agreement between the reconstructed state and the experimental data). Our target wave function is a collimated Gaussian beam from a single-mode fiber. The lens after the fiber is slightly displaced to create an aberrated wave front. This creates a complex wave function made from both real and imaginary parts. We reconstruct the wave function from the conventional direct measurement method using Eq. (1). The real and imaginary parts from a pixel-by-pixel raster scan are shown on the left column of Fig. 2 for a $N=12 \times 16=$ 192 dimensional Hilbert space. The real and imaginary parts of the wave function reconstructed from the CDM method using $N=192$ and $M / N=20 \%$ are shown on the middle column. It can be seen that the main features of the

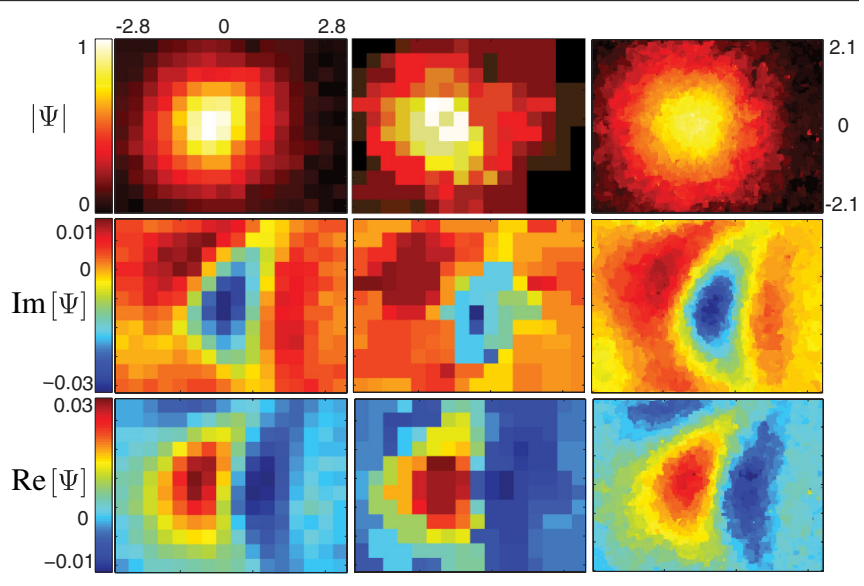

FIG. 2 (color online). The amplitude, imaginary, and real parts of an aberrated Gaussian state from experimental data. The left column presents data from a pixel-by-pixel scan of the state for $N=192$. The middle column shows the reconstructed wave front for $N=192$, and $M / N=20 \%$ of the total measurements from the CDM method. The right column demonstrates reconstruction for $N=19200$, and $M / N=20 \%$ of the total measurements. The transverse dimensions of the state are shown in millimeters.

state are retrieved with as few as $20 \%$ of the total number of measurements used in the left column. It should be emphasized that the minimum number of required measurements for an accurate reconstruction is proportional to the sparsity of the signal. Our algorithm uses sparsity with respect to the gradient transformation, according to Eq. (10). In order to achieve a more sparse signal, we have performed a fine grain measurement of the same state at the resolution of $N=120 \times 160=19200$. The wave function reconstructed from the CDM method using $M / N=20 \%$ is shown on the right column of Fig. 2 . Due to increased sparsity of the state in the larger Hilbert space, a very detailed reconstruction can be achieved with $20 \%$ of the total number of measurements.

To provide a quantitative comparison of the two methods we calculate the fidelity between the retrieved state $\left|\psi^{\prime}\right\rangle$ and the target state $|\psi\rangle$ from a full pixel-by-pixel scan as

$$
F\left(\left|\psi^{\prime}\right\rangle,|\psi\rangle\right)=\left|\left\langle\psi^{\prime} \mid \psi\right\rangle\right| .
$$

The results are shown in Fig. 3. The horizontal axis corresponds to the percentage of the measurements $(100 \times(M / N))$. The blue curve shows the fidelity of the state reconstructed with the CDM method. The red curve represents the average fidelity of the state reconstructed with Eq. (8) using the data from a partial pixel-by-pixel measurement of $M$ randomly chosen points. It is seen from the figure that the compressive method results in a drastic increase of fidelity for the first few measurements and gradually settles to a value close to 1 . As an example of the usefulness of the compressive method, a fidelity as high as $90 \%$ is achieved by performing only $25 \%$ of the measurements, while the conventional direct measurement needs 


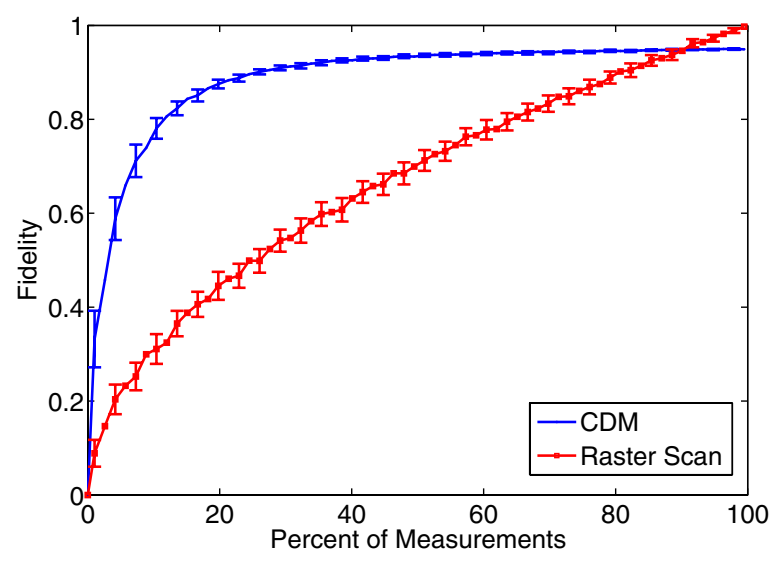

FIG. 3 (color online). The fidelity of the reconstructed state with the target wave function as a function of the percentage of the total measurements. The fidelity of the state reconstructed with CDM is shown in blue. The fidelity of the state reconstructed from a partial pixel-by-pixel scan with the same number of measurements is shown in red for comparison. The error bars represent standard deviation calculated from 100 repetitions of the experiment (error bars are shown at every second data point for visual clarity).

approximately $80 \%$ of all the measurements to achieve the same fidelity.

To further demonstrate the accuracy of our method we have used it to measure a custom state prepared using a phase mask depicting the letters $U$ and $R$ with a phase jump of $\pi / 2$. The phase mask is prepared via an additional spatial light modulator illuminated with the Gaussian beam from the laser and the state is imaged onto the second SLM, which is used for polarization rotation. Figure 4 shows the amplitude and the phase of the reconstructed state with $M / N=20 \%$ of the total measurements. Notice that while the amplitude is relatively uniform, the phase shows the letters $U$ and $R$ with a remarkable accuracy. It should be emphasized that the measurement of a state of such high dimensions is extremely time consuming via a pixel-bypixel scanning. In our approach, we perform a weak measurement on approximately half of all the pixels at each time. Due to this, the change in the state of the pointer (i.e., the polarization of the beam after the pinhole) is much more pronounced as compared to the conventional DM method where only one pixel would be weakly measured. The speed-up factor can be estimated considering that the strength of the signal measured in the laboratory is proportional to the value of the second term in Eq. (4). It is easy to check that the magnitude of this term is on average $\sqrt{N} / 2$ times larger in the case when half of the $Q_{m, j}$ are set to one. For the case of our experiment with $N=19200$, and $M / N=20 \%$, our approach provides a $\sim 350$-fold speed-up in the measurement procedure.

It should be emphasized that our specific experimental realization of the CDM method can be described using classical physics. The measured wave function in this case

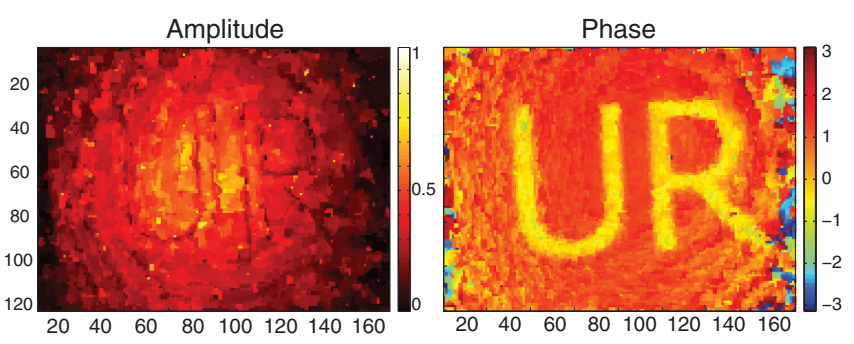

FIG. 4 (color online). The amplitude and phase of a Gaussian mode illuminating a custom phase mask (the initials of the University of Rochester). The data are reconstructed by the CDM method with $N=19200$, and $M / N=20 \%$ of the total measurements.

is the spatial mode of photons, which is equivalent to the electric field of paraxial light beams in the classical limit [37]. Since the experiment is designed to measure the spatial mode, it is insensitive to the number of excitations of the field (i.e., the number of photons). Subsequently, the results of the experiment would be identical for a source of single photons, heralded single photons, or a strong laser beam provided that they are prepared in the same spatial and polarization modes. However, the language of quantum mechanics provides a simpler description, with a broader range of applicability that includes fundamentally quantum mechanical states such as electron beams.

Recently, compressive sensing has been used to simultaneously measure the position and momentum distributions of photons with the aid of partially projective measurements [38]. This method requires parallel simultaneous measurements performed by a camera for characterizing probability distributions of conjugate variables. In contrast, the exclusive use of single-pixel detectors in our technique provides an alternative to inefficient and costly arrayed detectors for quantum optics [39] and terahertz applications [40]. Additionally, the use of a single-pixel detector is advantageous to parallel multipixel measurements in terms of the signal-to-noise ratio. Last but not least, we emphasize that the measurement of position and momentum probability distributions is not identical to the full measurement of the wave function. The measurement results from the method in Ref. [38] need to be processed using recursive numerical algorithms to retrieve the phase information, which is crucial for determination of the wave function. Such algorithms are proved to provide a unique solution only for specific sets of conditions [41].

Determining an unknown wave function is of fundamental importance in quantum mechanics. Despite many seminal contributions, in practice this task remains challenging, especially for high-dimensional states. The direct measurement approach, introduced by Lundeen $e t$ al., has provided a ground for meeting the high-dimensionality challenge [14]. Here, we combine the efficiency of compressive sensing with the simplicity of the direct measurement in determining the wave function of an a priori 
unknown state. Our experimental results demonstrate that a compressive variation of the direct measurement allows an accurate determination of a 192-dimensional state with a fidelity of $90 \%$ using only 25 percent of the measurements that are needed for a simple direct measurement approach. This method provides an easy means of characterizing high-dimensional systems in the labs. In addition, this technique can be used for classical applications such as wave front sensing.

We acknowledge helpful discussions with J. H. Eberly, B. Rodenburg, and Z. Shi. This work was supported by the DARPA/DSO InPho program, the U.S. Defense Threat Reduction Agency (Grant No. HDTRA1-10-1-0025), and the Canadian Excellence Research Chair (CERC) program. O.S.M.-L. also acknowledges support from the CONACyT. S. M. H. R. acknowledges financial support from NSF Grant No. PHY-1203931.

*mirhosse@optics.rochester.edu

[1] W. K. Wootters and W. H. Zurek, Nature (London) 299, 802 (1982).

[2] D. Dieks, Phys. Lett. 92A, 271 (1982).

[3] P. W. Milonni and M. L. Hardies, Phys. Lett. 92A, 321 (1982).

[4] B. Kanseri, T. Iskhakov, I. Agafonov, M. Chekhova, and G. Leuchs, Phys. Rev. A 85, 022126 (2012).

[5] M. Cramer, M. B. Plenio, S. T. Flammia, R. Somma, D. Gross, S. D. Bartlett, O. Landon-Cardinal, D. Poulin, and Y.-K. Liu, Nat. Commun. 1, 149 (2010).

[6] M. Hofheinz et al., Nature (London) 459, 546 (2009).

[7] K. J. Resch, P. Walther, and A. Zeilinger, Phys. Rev. Lett. 94, 070402 (2005).

[8] M. Beck, C. Dorrer, and I. A. Walmsley, Phys. Rev. Lett. 87, 253601 (2001).

[9] D. F. V. James, P. G. Kwiat, W. J. Munro, and A. G. White, Phys. Rev. A 64, 052312 (2001).

[10] M. G. Raymer, M. Beck, and D. F. McAlister, Phys. Rev. Lett. 72, 1137 (1994).

[11] D. T. Smithey, M. Beck, M. G. Raymer, and A. Faridani, Phys. Rev. Lett. 70, 1244 (1993).

[12] K. Vogel and H. Risken, Phys. Rev. A 40, 2847 (1989).

[13] M. Agnew, J. Leach, M. McLaren, F. S. Roux, and R. W. Boyd, Phys. Rev. A 84, 062101 (2011).

[14] J. S. Lundeen, B. Sutherland, A. Patel, C. Stewart, and C. Bamber, Nature (London) 474, 188 (2011).

[15] J. Z. Salvail, M. Agnew, A. S. Johnson, E. Bolduc, J. Leach, and R. W. Boyd, Nat. Photonics 7, 316 (2013).
[16] M. Malik, M. Mirhosseini, M. P. J. Lavery, J. Leach, M. J. Padgett, and R. W. Boyd, Nat. Commun. 5, 3115 (2014).

[17] J. Fischbach and M. Freyberger, Phys. Rev. A 86, 052110 (2012).

[18] S. Wu, Sci. Rep. 3, 1 (2013).

[19] A. Di Lorenzo, Phys. Rev. Lett. 110, 010404 (2013).

[20] L. Maccone and C. C. Rusconi, Phys. Rev. A 89, 022122 (2014).

[21] A. Shabani, R. L. Kosut, M. Mohseni, H. Rabitz, M. A. Broome, M. P. Almeida, A. Fedrizzi, and A. G. White, Phys. Rev. Lett. 106, 100401 (2011).

[22] G. A. Howland and J. C. Howell, Phys. Rev. X 3, 011013 (2013).

[23] W.-T. Liu, T. Zhang, J.-Y. Liu, P.-X. Chen, and J.-M. Yuan, Phys. Rev. Lett. 108, 170403 (2012).

[24] D. Gross, Y.-K. Liu, S. T. Flammia, S. Becker, and J. Eisert, Phys. Rev. Lett. 105, 150401 (2010).

[25] O. Katz, Y. Bromberg, and Y. Silberberg, Appl. Phys. Lett. 95, 131110 (2009).

[26] M. F. Duarte, M. A. Davenport, D. Takhar, J. N. Laska, T. Sun, K. F. Kelly, and R. G. Baraniuk, IEEE Signal Process. Mag. 25, 83 (2008).

[27] Y. Aharonov, D. Z. Albert, and L. Vaidman, Phys. Rev. Lett. 60, 1351 (1988).

[28] R. Jozsa, Phys. Rev. A 76, 044103 (2007).

[29] J. Romberg, IEEE Signal Process. Mag. 25, 14 (2008).

[30] S. L. Campbell and C. D. Meyer, Generalized Inverses of Linear Transformations, Vol. 56 (SIAM, Philadelphia, 2009).

[31] E. Candes and J. Romberg, Inverse Probl. 23, 969 (2007).

[32] P. Zerom, K. W. C. Chan, J. C. Howell, and R. W. Boyd, Phys. Rev. A 84, 061804 (2011).

[33] O. S. Magaña-Loaiza, G. A. Howland, M. Malik, J. C. Howell, and R. W. Boyd, Appl. Phys. Lett. 102, 231104 (2013).

[34] M. Mirhosseini, M. Malik, M. Lavery, J. Leach, M. Padgett, and R. W. Boyd, Frontiers in Optics, FW4A.3 (2012).

[35] I. Moreno, J. L. Martinez, and J. A. Davis, Appl. Opt. 46, 881 (2007).

[36] C. Li, W. Yin, and Y. Zhang, in "User's Guide for TVAL3: TV Minimization by Augmented Lagrangian and Alternating Direction Algorithms," Rice University (2010).

[37] C. Bamber, B. Sutherland, A. Patel, C. Stewart, and J. S. Lundeen, Opt. Express 20, 2034 (2012).

[38] G. A. Howland, J. Schneeloch, D. J. Lum, and J. C. Howell, Phys. Rev. Lett. 112, 253602 (2014).

[39] R. H. Hadfield, Nat. Photonics 3, 696 (2009).

[40] W. L. Chan, M. L. Moravec, R. G. Baraniuk, and D. M. Mittleman, Opt. Lett. 33, 974 (2008).

[41] J. R. Fienup, Appl. Opt. 21, 2758 (1982). 